\title{
Comparative clinicopathological changes in buffalo and cattle following infection by Pasteurella multocida B:2
}

\begin{abstract}
Haemorrhagic septicaemia (HS) is an acute, septicaemic disease of cattle and buffalo of Asia and Africa caused by Pasteurella multocida B:2 or E:2. Buffaloes are believed to be more susceptible than cattle. In this study, 9 buffaloes of 8 months old were divided equally into 3 groups (Groups 1, 3, 5). Similarly, 9 cattle of 8 months old were equally divided into 3 groups (Groups 2, 4, 6). Animals of Groups 1 and 2 were inoculated with PBS while Groups 3 and 4 were inoculated subcutaneously with $10(5) \mathrm{cfu} / \mathrm{ml}$ of P. multocida B:2. Animals of Groups 5 and 6 were inoculated intranasally with the same inoculum. Both buffaloes and cattle that were inoculated subcutaneously succumbed to the infection at $16 \mathrm{~h}$ and $18 \mathrm{~h}$, respectively. Two buffaloes that were inoculated intranasally (Group 5) succumbed at $68 \mathrm{~h}$ while the remaining cattle and buffaloes survived the 72-h study period. Endotoxin was detected in the blood of infected cattle (Group 4) and buffaloes (Groups 3 and 5) prior to the detection of P. multocida B:2 in the blood. The endotoxin was detected in the blood of buffaloes of Group 3 and cattle of Group 4 at 0.5 h post-inoculation while buffaloes of Group 5 and cattle of Group 6 at $1.5 \mathrm{~h}$. On the other hand, bacteraemia was detected at $2.5 \mathrm{~h}$ in buffaloes of Group 3 and cattle of Group 4 and at $12 \mathrm{~h}$ in buffaloes of Group 5 and cattle of Group 6. Affected cattle and buffaloes showed lesions typical of haemorrhagic septicaemia. These included congestion and haemorrhages in the organs of respiratory, gastrointestinal and urinary tracts with evidence of acute inflammatory reactions. The severity of gross and histopathology lesions in cattle and buffalo calves that succumbed to the infection showed insignificant $(\mathrm{p}>0.05)$ difference. However, inoculated buffalo and cattle that survived the infection showed significantly $(\mathrm{p}<0.05)$ less severe gross and histopathological changes than those that succumbed. In general, cattle are more resistant to intranasal infection by $\mathrm{P}$. multocida B:2 than buffaloes.
\end{abstract}

Keyword: Bacteraemia; Buffalo; Cattle; Endotoxaemia; Pasteurella multocida B:2 